\title{
The 2018 X-Ray and Radio Outburst of Magnetar XTE J1810-197
}

\author{
E. V. Gotthelf ${ }^{1}$ (1) , J. P. Halpern ${ }^{1}$, J. A. J. Alford ${ }^{1}$, T. Mihara ${ }^{2}$, H. Negoro ${ }^{3}$, N. Kawai ${ }^{4}\left(\mathbb{D}\right.$, S. Dai $^{5}$, M. E. Lower ${ }^{6}\left(\mathbb{1}\right.$, S. Johnston $^{5}$, \\ M. Bailes ${ }^{6}$ (D), S. Osłowski ${ }^{6}$, F. Camilo ${ }^{7}$ (D), H. Miyasaka ${ }^{8}$ (D), and K. K. Madsen ${ }^{8}$ (iD \\ ${ }^{1}$ Columbia Astrophysics Laboratory, Columbia University, 550 West 120th Street, New York, NY 10027, USA \\ ${ }^{2}$ High Energy Astrophysics Laboratory, RIKEN, 2-1 Hirosawa, Wako, Saitama 351-0198, Japan \\ ${ }^{3}$ Department of Physics, Nihon University, 1-8 Kanda-Surugadai, Chiyoda-ku, Tokyo 101-8308, Japan \\ ${ }^{4}$ Department of Physics, Tokyo Institute of Technology, 2-12-1 Ookayama, Meguro-ku, Tokyo 152-8551, Japan \\ ${ }^{5}$ CSIRO Astronomy and Space Science, Australia Telescope National Facility, P.O. Box 76, Epping, NSW 1710, Australia \\ ${ }^{6}$ Centre for Astrophysics and Supercomputing, Swinburne University of Technology, VIC 3122, Australia \\ ${ }^{7}$ South African Radio Astronomy Observatory, Observatory 7925, South Africa \\ ${ }^{8}$ Cahill Center for Astronomy and Astrophysics, California Institute of Technology, Pasadena, CA 91125, USA \\ Received 2019 February 21; revised 2019 March 14; accepted 2019 March 15; published 2019 April 2
}

\begin{abstract}
We present the earliest X-ray observations of the 2018 outburst of XTE J1810-197, the first outburst since its 2003 discovery as the prototypical transient and radio-emitting anomalous X-ray pulsar (AXP). The Monitor of Allsky X-ray Image (MAXI) detected XTE J1810-197 immediately after a November 20-26 visibility gap, contemporaneous with its reactivation as a radio pulsar, first observed on December 8. On December 13 the Nuclear Spectroscopic Telescope Array (NuSTAR) detected X-ray emission up to at least $30 \mathrm{keV}$, with a spectrum well-characterized by a blackbody plus power-law model with temperature $k T=0.74 \pm 0.02 \mathrm{keV}$ and photon index $\Gamma=4.4 \pm 0.2$ or by a two-blackbody model with $k T=0.59 \pm 0.04 \mathrm{keV}$ and $k T=1.0 \pm 0.1 \mathrm{keV}$, both including an additional power-law component to account for emission above $10 \mathrm{keV}$, with $\Gamma_{h}=-0.2 \pm 1.5$ and $\Gamma_{h}=1.5 \pm 0.5$, respectively. The latter index is consistent with hard X-ray flux reported for the nontransient magnetars. In the $2-10 \mathrm{keV}$ bandpass, the absorbed flux is $2 \times 10^{-10} \mathrm{erg} \mathrm{s}^{-1} \mathrm{~cm}^{-2}$, a factor of 2 greater than the maximum flux extrapolated for the 2003 outburst. The peak of the sinusoidal X-ray pulse lags the radio pulse by $\approx 0.13$ cycles, consistent with their phase relationship during the 2003 outburst. This suggests a stable geometry in which radio emission originates on magnetic field lines containing currents that heat a spot on the neutron star surface. However, a measured energy-dependent phase shift of the pulsed X-rays suggests that all X-ray emitting regions are not precisely coaligned.
\end{abstract}

Key words: pulsars: general - pulsars: individual (XTE J1810-197) - stars: neutron - X-rays: stars

\section{Introduction}

Magnetars are neutron star (NS) pulsars whose X-ray luminosity can greatly exceed their spin-down power. Unlike for the canonical rotation-powered radio pulsars, the luminosity of magnetars is thought to be supplied by the decay of their large magnetic fields, typically $\approx 10^{14-15} \mathrm{G}$. The dipole field components result in rapid spin-down and long rotation periods in the 0.3-12 s range. The discovery of XTE J1810-197 (Gotthelf et al. 2004; Ibrahim et al. 2004) marked a turning point in the study of magnetars. Until then, the known magnetars comprised four transient soft gamma-ray repeaters (SGRs), and five persistent anomalous X-ray pulsars (AXPs) detected by UHURU, Einstein, or ROSAT. The two classes shared similar magnetic field strengths and spin periods but had different long-term histories. The SGRs had rare, violent outbursts, while the AXPs were fairly steady emitters. See recent reviews by Kaspi \& Beloborodov (2017), Esposito et al. (2018), and Coti Zelati et al. (2018).

XTE J1810-197 was the first recognized transient AXP, detected in outburst in 2003 with a period of $5.54 \mathrm{~s}$ at a flux level $\sim 140$ times higher than its quiescent state as a previously anonymous ROSAT source. It decayed roughly as a $\tau \approx$ 280 day exponential. A Very Large Array (VLA) survey in 2004 serendipitously detected a point source at the position of XTE J1810-197 (Halpern et al. 2005); subsequently pulsed radio emission was searched for and detected for the first time in a magnetar (Camilo et al. 2006). Until then it had been theorized that high magnetic fields inherently suppressed radio pulsations. On the contrary, XTE J1810-197 was a bright transient radio pulsar, but with a flatter spectrum than ordinary pulsars, such that it was the brightest NS known at frequencies above $20 \mathrm{GHz}$. Three more transient magnetars have been detected as radio pulsars with similar properties (Camilo et al. 2007a; Levin et al. 2010; Eatough et al. 2013; Shannon \& Johnston 2013).

Meanwhile, short SGR-like bursts had also been discovered from AXPs (Gavriil et al. 2002; Kaspi et al. 2003), reinforcing the connection between the two magnetar classes. Similar bursts were detected during the decay of XTE J1810-197 (Woods et al. 2005). Beginning with the launch of Swift/BAT, transient magnetars have been discovered on a regular basis, each outburst signaled by one or more short SGR-like burst. There are now a total of 23 confirmed magnetars. ${ }^{9}$ Interestingly, no new persistent ones have been discovered since 2007, suggesting that most magnetars are transient.

XTE J1810-197 provides a crucial probe of NS surface physics because of its relatively close distance (3-4 kpc Durant \& van Kerkwijk 2006; Minter et al. 2008) and continuing quiescent emission, not detected in most other transient magnetars. This allows the evolving spectrum and pulse profiles of XTE J1810-197 to be modeled (Perna \& Gotthelf 2008; Albano et al. 2010; Bernardini et al. 2011), mapping the magnetar's cooling and shrinking surface thermal hot spots,

\footnotetext{
9 http://www.physics.mcgill.ca/ pulsar/magnetar/main.html
} 
presumably powered by currents along the untwisting magnetic field-line bundles (“j-bundles," Beloborodov 2009, 2013).

In the following sections we present the first X-ray observations of XTE J1810-197 during its 2018 outburst. Monitor of All-sky $X$-ray Image (MAXI) all-sky monitoring data constrains the epoch of the outburst, and a timely Nuclear Spectroscopic Telescope Array $(\mathrm{NuSTAR})$ observation within $\approx 3$ weeks of the onset characterizes its early spectrum in the $3-30 \mathrm{keV}$ band. A comparison of the X-ray pulse phase with a contemporaneous radio pulse observation is also made. We discuss our results in the context of the previous outburst and future expectations.

\section{X-Ray Observations}

The reports of intense radio emission (Desvignes et al. 2018; Lower et al. 2018; Lyne et al. 2018) and enhanced X-ray flux (Mihara et al. 2018) from XTE J1810-197 signaled a new outburst from this magnetar that had occurred sometime between 2018 October 26 and December 8 (radio) and between 2018 November 20-26 (X-ray; this work). Based on the Lyne et al. (2018) discovery, we initiated a NuSTAR Director's Discretionary Time observation of the magnetar. Preliminary NUSTAR results were reported in Gotthelf et al. (2018) using a subset $(\sim 50 \%)$ of the data. In the current work, we analyze the complete NUSTAR observation of XTE J1810-197, along with the $M A X I$ all-sky monitoring light curve, to determine its early outburst spectral and temporal properties.

In the following study, all spectra for XTE J1810-197 are fitted using XSPEC v12.10.0c software (Arnaud 1996) with the column density characterized by the default TBabs absorption model. Spectral uncertainties are computed for the $90 \%$ confidence level for two interesting parameters unless otherwise noted. For the timing analysis, photons arrival times were converted to the solar system barycenter using the radio coordinates and the JPL DE200 planetary ephemeris.

\subsection{MAXI Results}

The MAXI observatory (Matsuoka et al. 2009) is attached to the International Space Station (ISS) and scans the sky 16 times a day during its 92 minute orbit, using the Gas Slit Camera (GSC; Mihara et al. 2011) to build up images in the $2-30 \mathrm{keV}$ band. Although XTE J1810-197 is not a MAXI cataloged object (Hori et al. 2018), the region containing the magnetar was observed with the GSC as part of the MAXI Nova-Alert System program (Negoro et al. 2016). The region was observed before and after the reported radio event for $\approx 40-140$ s per scan, with data gaps due to Earth-block, ISS structure obscuration, SAA passages, and periods of high particle background. A light curve at the position of XTE J1810-197 was extracted from stacked 1 day image scans using a 1.6 radius aperture, excluding two slightly overlapping sources. Starting on November 26 (MJD 58448), we detect a significant increase in the 1 day count rates, following a 6 day gap that lacked reliable image data. During the interval MJD $58450-58498$, the average $2-10 \mathrm{keV}$ rate increased to $0.0195 \pm 0.0013 \mathrm{~s}^{-1} \mathrm{~cm}^{-2}$, compared to the pre-outburst rate of $0.0012 \pm 0.0062 \mathrm{~s}^{-1} \mathrm{~cm}^{-2}$. Figure 1 displays the $2-10 \mathrm{keV}$ light curve in energy flux units, rebinned to obtain at least a $5 \sigma$ detection over a maximum time span of 4 days. MAXI count rates were converted to flux units using the PIMMS ${ }^{10}$ software,

\footnotetext{
${ }^{10}$ Portable, Interactive Multi-Mission Simulator; https://heasarc.gsfc.nasa. gov/docs/software/tools/pimms.html.
}

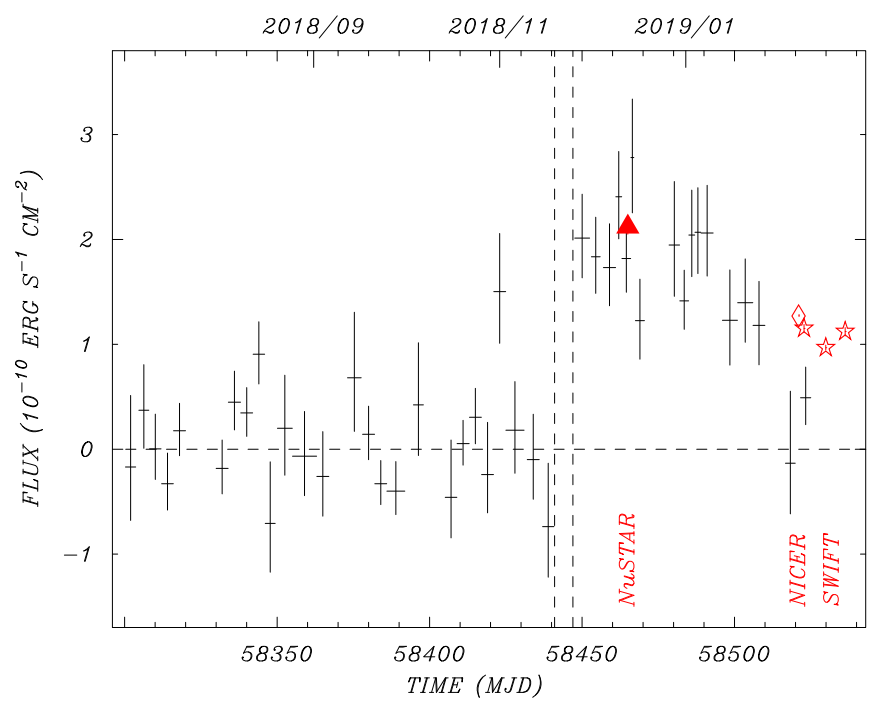

Figure 1. MAXI 2-10 keV light curve of XTE J1810-197 using a variable binning scheme (see the text). The vertical lines bound the possible time of outburst. The decrease in flux over time since outburst is evident and consistent with flux measurements obtained with NUSTAR (triangle; this work), NICER (diamond; Güver et al. 2019), and Swift (stars). The latter were obtained from spectra generated from reprocessed Swift archival data fitted with a blackbody model in the $1-5 \mathrm{keV}$ range.

for a blackbody temperature of $0.7 \mathrm{keV}$, estimated from the average $4-10 \mathrm{keV} / 2-4 \mathrm{keV}$ hardness ratio during the MJD 58450-58498 interval. We conclude that XTE J1810-197 became active in X-rays sometime between November 20-26.

\subsection{NuSTAR Observation}

We obtained a $22 \mathrm{hr}$ observation of XTE J1810-197 starting on UT 2018 December 13 at 03:10:21 UT. At this time, NUSTAR was the only X-ray mission capable of imaging the source so close $\left(12^{\circ}\right)$ to the Sun. A single source is detected in the field of view, with a count rate of $4.8 \mathrm{~s}^{-1}$ and flux up to at least $30 \mathrm{keV}$ in the $3-79 \mathrm{keV}$ band. Recovery of the expected $5.54 \mathrm{~s}$ NS spin period identifies the source as XTE J1810-197 (see Section 2.3.3).

NUSTAR consists of two coaligned X-ray telescopes, with corresponding focal plane detector modules FPMA and FPMB, each of which is composed of a $2 \times 2$-node $\mathrm{CdZnTe}$ sensor array (Harrison et al. 2013). These are sensitive to X-rays in the 3-79 keV band, with a characteristic spectral resolution of $400 \mathrm{eV}$ FWHM at $10 \mathrm{keV}$. The multinested foil mirrors provide $18^{\prime \prime}$ FWHM (58" HPD) imaging resolution over a $12 ! 2 \times 12 ! 2$ field of view (Harrison et al. 2013). The nominal timing accuracy of NUSTAR is $\sim 2 \mathrm{~ms}$ rms, after correcting for drift of the on-board clock, with the absolute timescale shown to be better than $<3 \mathrm{~ms}$ (Mori et al. 2014; Madsen et al. 2015). This is more than sufficient to resolve the X-ray signal from XTE J1810-197 and to compare it to the radio pulse.

The reconstructed NUSTAR coordinates are nominally accurate to $7 . ! 5$ (90\% confidence level); however, a full aspect reconstruction is not possible for this observation due to the proximity of the Sun to the target. For this pointing, star tracker \#4, which is coaligned with the X-ray optics, was not available to recover the absolute aspect or to remove the $2^{\prime}$ image blur due to telescope mast motion. During the orbit, a cyclic combination of the other three star trackers was used to determine the attitude. As outlined below, we analyze data 
from each star tracker combination separately to help compensate for the mast motion.

This work uses the data made available via the NuSTAR ToO web page, which was processed and analyzed using FTOOLS 22Oct2018_V6.25 (NUSTARDAS 06Jul17_V1.8.0) with $N u S$ TAR Calibration Database (CALDB) files of 2016 July 6. Event files were generated for each star tracker configuration using the split_sc option in nupipeline. The processed data was uncontaminated by solar wind events and provides a total of $36 \mathrm{ks}$ of good exposure time.

\subsection{The NuSTAR Analysis}

To generate a NUSTAR spectrum for XTE J1810-197, we analyzed data separately from the five available star tracker configurations for this observation, to allow for a noticeable $\sim 2^{\prime}$ shift in the source location on the focal plane, periodic on an orbit timescale. However, these star tracker configurations break each orbit into five pieces, adjacent in time, and the drift is not noticeable over these relatively short intervals. Thus, we computed the source centroid on a per orbit, per star tracker configuration basis, to center the extraction region based on the drift. This allows us to use a smaller extraction aperture of 0.75 radius as compared to the $2^{\prime}$ radius of our previous analysis (Gotthelf et al. 2018), greatly reducing the relative background contribution in the source aperture at higher energies.

Although the NuSTAR image of XTE J1810-197 contained no other sources, there are regions on the focal plane contaminated by stray light. In particular, stray light from nearby GX $9+1$ partially overlaps the source region in the FPMA image. For each detector we determined a suitable background region and accounted for the contaminating fraction of stray light in the source region, where appropriate. In all, for each FPM, we generated 67 sets of source and background spectral files along with their response functions. The final summed/averaged FMPA and FPMB spectra and their response files were grouped to include at least 100 counts per channel and fitted to several spectral models of interest, as described below. For these fits, the absorption column is not well constrained in the NuSTAR energy band and is held fixed to a nominal $N_{\mathrm{H}}=1.0 \times 10^{22} \mathrm{~cm}^{-2}$.

\subsubsection{The $<10 \mathrm{keV}$ NuSTAR Spectrum}

To compare to the early results for the 2003 outburst of XTE J1810-197, obtained on 2003 September 8 using XMMNewton (Halpern \& Gotthelf 2005), we fit the NuSTAR spectrum in the restricted $3-10 \mathrm{keV}$ band. A blackbody plus power-law model yields a best-fit temperature $k T=0.72 \pm 0.02 \mathrm{keV}$ and photon index $\Gamma=4.2 \pm 0.2$, with $\chi_{\nu}^{2}=1.03$ for 234 degrees of freedom (DoF), comparable, but hotter and harder than that found for the 2003 outburst $(k T=0.67 \pm 0.02 \mathrm{keV}, \Gamma=3.8 \pm 0.2)$, consistent with the present detection being at an earlier phase. The absorbed flux of $F(2-10 \mathrm{keV})=(2.13 \pm 0.06) \times$ $10^{-10} \mathrm{erg} \mathrm{s}^{-1} \mathrm{~cm}^{-2}$, however, is a factor of $\approx 2$ greater than the projected maximum flux of $F(2-10 \mathrm{keV})=(0.8-1.1) \times$ $10^{-10} \mathrm{erg} \mathrm{s}^{-1} \mathrm{~cm}^{-2}$ for the 2003 outburst (Gotthelf \& Halpern 2007). For the estimated distance of $3.5 \mathrm{kpc}$ to XTE J1810-197, the higher bolometric luminosity of $L_{\mathrm{bol}}=2.6 \times 10^{35} \mathrm{erg} \mathrm{s}^{-1}$ implies a blackbody area of $A_{\mathrm{BB}}=9.6 \times 10^{11} \mathrm{~cm}^{2}$, substantially larger than that measured $\sim 8$ months into the previous outburst.

In contrast, a perhaps more realistic two-blackbody model, also fitted in the $3-10 \mathrm{keV}$ band, yields much higher temperatures of $k T_{\text {hot }}=1.19 \pm 0.08 \mathrm{keV}$ and $k T_{\text {warm }}=$ $0.62 \pm 0.02 \mathrm{keV}\left(\chi_{\nu}^{2}=1.03\right.$ for $\left.234 \mathrm{DoF}\right)$ as compared to the prior outburst $(k T=0.68 \pm 0.02 \mathrm{keV}$ and $k T=0.26 \pm$ $0.02 \mathrm{keV})$. The absorbed flux is $F(2-10 \mathrm{keV})=$ $(1.9 \pm 0.3) \times 10^{-10}$ for this model. The bolometric luminosities are $L_{\mathrm{hot}}=6.5 \times 10^{35} \mathrm{erg} \mathrm{s}^{-1}$ and $L_{\mathrm{warm}}=4.6 \times$ $10^{35} \mathrm{erg} \mathrm{s}^{-1}$, with implied areas of $A_{\text {hot }}=3.2 \times 10^{10} \mathrm{~cm}^{2}$ and $A_{\text {warm }}=2.9 \times 10^{12} \mathrm{~cm}^{2}$. Another physically motivated model is the Comptonized blackbody, with best-fit parameters $k T=0.632 \pm 0.008 \mathrm{keV}$ and $\alpha=2.71 \quad\left(\chi_{\nu}^{2}=1.12\right.$ for 238 DoF). Specifically, we use the model described in Halpern et al. (2008), where $\alpha \equiv-\ln \left(\tau_{\text {es }}\right) / \ln (A)$ is the $\log$ ratio of the scattering optical depth $\tau_{\text {es }}$ over the mean amplification $A$ of photon energy per scattering, valid for $\tau_{\text {es }} \ll 1$ (Rybicki \& Lightman 1986).

\subsubsection{The 3-30 keV NuSTAR Spectrum}

The low background contamination of the NUSTAR data set allows us to model the spectrum up to $30 \mathrm{keV}$ before running out of source photons. None of the two-component models alone can account for significant emission evident above $10 \mathrm{keV}$. To characterize this nonthermal emission we fit an additional power-law component to both the blackbody plus power-law model and the two-blackbody model described above. As shown in Figure 2, this yields an excellent fit for both models. The resulting best-fit parameters, presented in Table 1, are comparable to those obtained with the $3-10 \mathrm{keV}$ fits, unchanged within their mutual $90 \%$ confidence levels. We note that the two-blackbody model yield a photon index of $\Gamma_{h}=1.5 \pm 0.5$ for the added power-law component, a value typical of the nontransient magnetars. For the blackbody plus power-law model, however, the index is not well constrained and yields an index of $\Gamma_{h}=-0.2 \pm 1.5$, much flatter than might be expected (see Enoto et al. 2017).

We are confident in the need for an additional spectral component to characterize the $>10 \mathrm{keV}$ emission, as both FPM detectors yield consistent results when fitted independently, despite the greater contamination in the FPMA spectrum due to stray light from GX9+1, as is evident in Figure 3.

\subsubsection{Pulsar Timing}

To search for the expected pulsar signal from XTE J1810 -197 , we extracted and merged barycentered photons from both FPMs using a 0!75 radius aperture centered on the source, again compensating for the telescope mast motion as described above. Using the $Z_{1}^{2}$ statistic, we recover a highly significant signal with $P=5.5414479(34)$ s at MJD 58465.14 in the $3-10 \mathrm{keV}$ energy range, leaving no doubt as to the identity of the NuSTAR source, despite its poor aspect. The resulting pulse profile is nearly sinusoidal in shape, similar to that recorded by $X M M-N e w t o n$ during the previous outburst. However, the pulse modulation, determined by fitting a sinusoidal model to the background subtracted pulse profile, is lower by half (e.g., $21 \%$ versus $46 \%$ at $3 \mathrm{keV}$ ). The modulation is defined here as the ratio of the pulsed flux to the total flux in the pulse profile.

The pulse profile in the $3-5 \mathrm{keV}$ and $5-10 \mathrm{keV}$ bands is shown in Figure 4 (left) to highlight the clear shift in phase ( $\Delta \phi \approx 0.1$ ) between the two bands, accounting for at least some of the asymmetry in the broadband pulse profile. This is most evident in Figure 4 (right), which displays the phase as a function of energy, suggesting that the emission components 

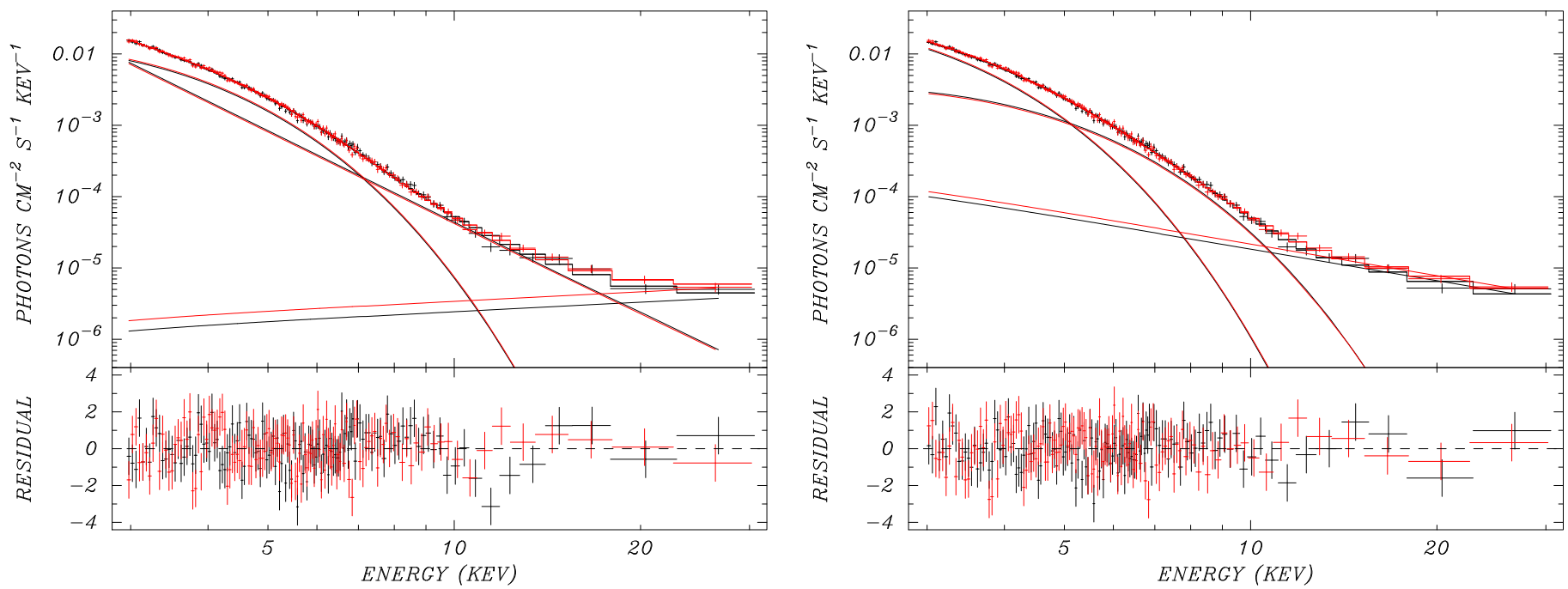

Figure 2. NUSTAR 3-30 keV spectrum of XTE J1810-197 obtained during its 2018 December outburst fitted to the blackbody plus power-law model (left) or twoblackbody model (right) described in the text. Both models include an additional power-law component to characterize the hard $>10 \mathrm{keV}$ flux. The top panel shows the unfolded data (crosses) and fitted model (solid lines) collected by the two focal plane modules FPMA (black) and FPMB (red), along with their spectral components. The lower panel shows the residual between the data and the model, in units of sigma.

Table 1

NUSTAR 3-30 keV Spectral Fit Results

\begin{tabular}{|c|c|}
\hline$\overline{\text { Model }}$ & Parameter \\
\hline \multicolumn{2}{|c|}{ Two-blackbody Model with Hard Power-law } \\
\hline$N_{\mathrm{H}}\left(10^{22} \mathrm{~cm}^{-2}\right)$ & 1.0 (fixed) \\
\hline$k T_{1}(\mathrm{keV})$ & $0.59 \pm 0.04$ \\
\hline$k T_{2}(\mathrm{keV})$ & $1.0 \pm 0.1$ \\
\hline$\Gamma_{h}$ & $1.5 \pm 0.5$ \\
\hline BB1 Flux $(2-10 \mathrm{keV})^{\mathrm{a}}$ & $1.4 \times 10^{-10}$ \\
\hline BB2 Flux $(2-10 \mathrm{keV})^{\mathrm{a}}$ & $5.3 \times 10^{-11}$ \\
\hline PL Flux $(2-10 \mathrm{keV})^{\mathrm{a}}$ & $3.4 \times 10^{-12}$ \\
\hline PL Flux $(2-30 \mathrm{keV})^{\mathrm{a}}$ & $8.1 \times 10^{-12}$ \\
\hline Total Flux $(2-10 \mathrm{keV})^{\mathrm{a}}$ & $1.9 \times 10^{-10}$ \\
\hline$L_{\mathrm{BB} 1}(\mathrm{bol})\left(\mathrm{erg} \mathrm{s}^{-1}\right)^{\mathrm{b}}$ & $4.3 \times 10^{35}$ \\
\hline$L_{\mathrm{BB} 2}(\mathrm{bol})\left(\mathrm{erg} \mathrm{s}^{-1}\right)^{\mathrm{b}}$ & $1.0 \times 10^{35}$ \\
\hline BB1 Area $\left(\mathrm{cm}^{2}\right)$ & $3.4 \times 10^{12}$ \\
\hline BB2 Area $\left(\mathrm{cm}^{2}\right)$ & $9.8 \times 10^{10}$ \\
\hline$\chi_{\nu}^{2}(\mathrm{DoF})$ & $1.04(250)$ \\
\hline \multicolumn{2}{|c|}{ Power-law + Blackbody Model with Hard Power-law } \\
\hline$N_{\mathrm{H}}\left(10^{22} \mathrm{~cm}^{-2}\right)$ & 1.0 (fixed) \\
\hline$k T_{\mathrm{BB}}(\mathrm{keV})$ & $0.74 \pm 0.02$ \\
\hline$\Gamma_{s}$ & $4.4 \pm 0.2$ \\
\hline$\Gamma_{h}$ & $-0.2 \pm 1.5$ \\
\hline BB Flux $(2-10 \mathrm{keV})^{\mathrm{a}}$ & $1.0 \times 10^{-10}$ \\
\hline $\mathrm{PL}_{\mathrm{s}}$ Flux $(2-10 \mathrm{keV})^{\mathrm{a}}$ & $1.2 \times 10^{-10}$ \\
\hline $\mathrm{PL}_{\mathrm{h}}$ Flux $(2-10 \mathrm{keV})^{\mathrm{a}}$ & $2.5 \times 10^{-13}$ \\
\hline $\mathrm{PL}_{\mathrm{h}}$ Flux $(2-30 \mathrm{keV})^{\mathrm{a}}$ & $3.1 \times 10^{-12}$ \\
\hline Total Flux $(2-10 \mathrm{keV})^{\mathrm{a}}$ & $2.2 \times 10^{-10}$ \\
\hline$L_{\mathrm{BB}}(\mathrm{bol})\left(\mathrm{erg} \mathrm{s}^{-1}\right)^{\mathrm{b}}$ & $2.5 \times 10^{35}$ \\
\hline BB Area $\left(\mathrm{cm}^{2}\right)$ & $8.0 \times 10^{11}$ \\
\hline$\chi_{\nu}^{2}(\mathrm{DoF})$ & $1.10(250)$ \\
\hline
\end{tabular}

Notes. Joint fits to FPMA and FPMB spectra with independent model normalizations. Uncertainties are $90 \%$ confidence for three interesting parameters.

${ }^{a}$ Absorbed flux in units of erg $\mathrm{cm}^{-2} \mathrm{~s}^{-1}$, averaged FPMA and FPMB values.

${ }^{\mathrm{b}}$ Luminosity is computed for a distance of $3.5 \mathrm{kpc}$.

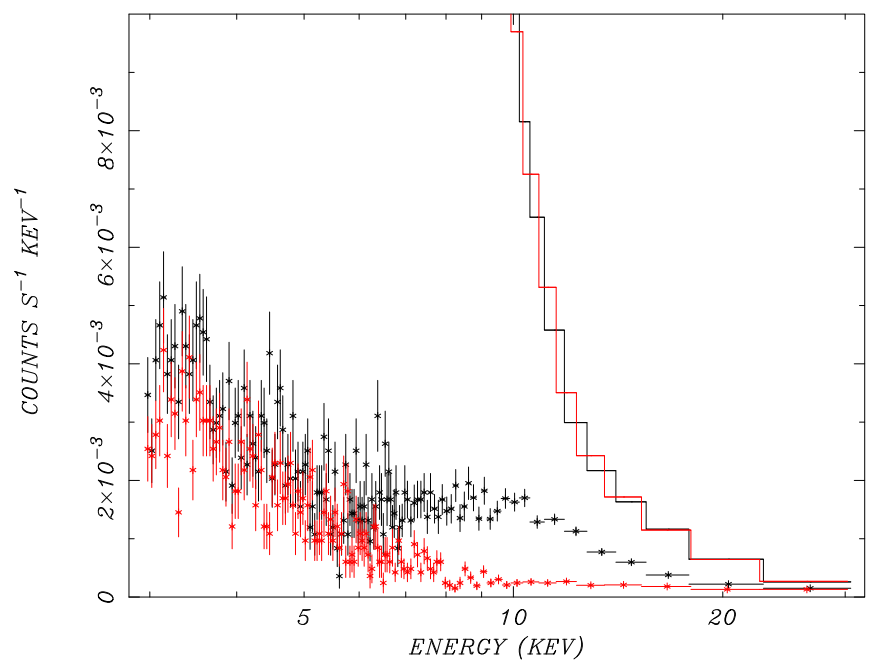

Figure 3. Best-fit blackbody plus power-law model (solid line) in the 3-30 kev band (see Figure 2) compared with the background contribution (crosses), scaled linearly. The background rate is negligible below $10 \mathrm{keV}$ and nowhere exceeds the source rate for all spectral channels in the full 3-30 keV energy range.

are not strictly coaxial. This figure also shows that the pulse modulation increases linearly with energy, turning over above $\sim 7 \mathrm{keV}$, notably near the spectral component cross-over energy in Figure 2. Within the short NUSTAR exposure, we find no evidence for bursts on second timescales, as were detected by RXTE during the earlier outburst (Woods et al. 2005).

Radio pulse observations bracketing the epoch of the NuSTAR observation were obtained by Dai et al. (2019) using the Parkes Telescope with the Ultra-Wideband Low receiver system (UWL; G. Hobbs et al. 2019, in preparation), covering a frequency range of $704-4032 \mathrm{MHz}$, and by $\mathrm{M}$. Lower et al. (2019, in preparation) using the upgraded Molonglo Observatory 

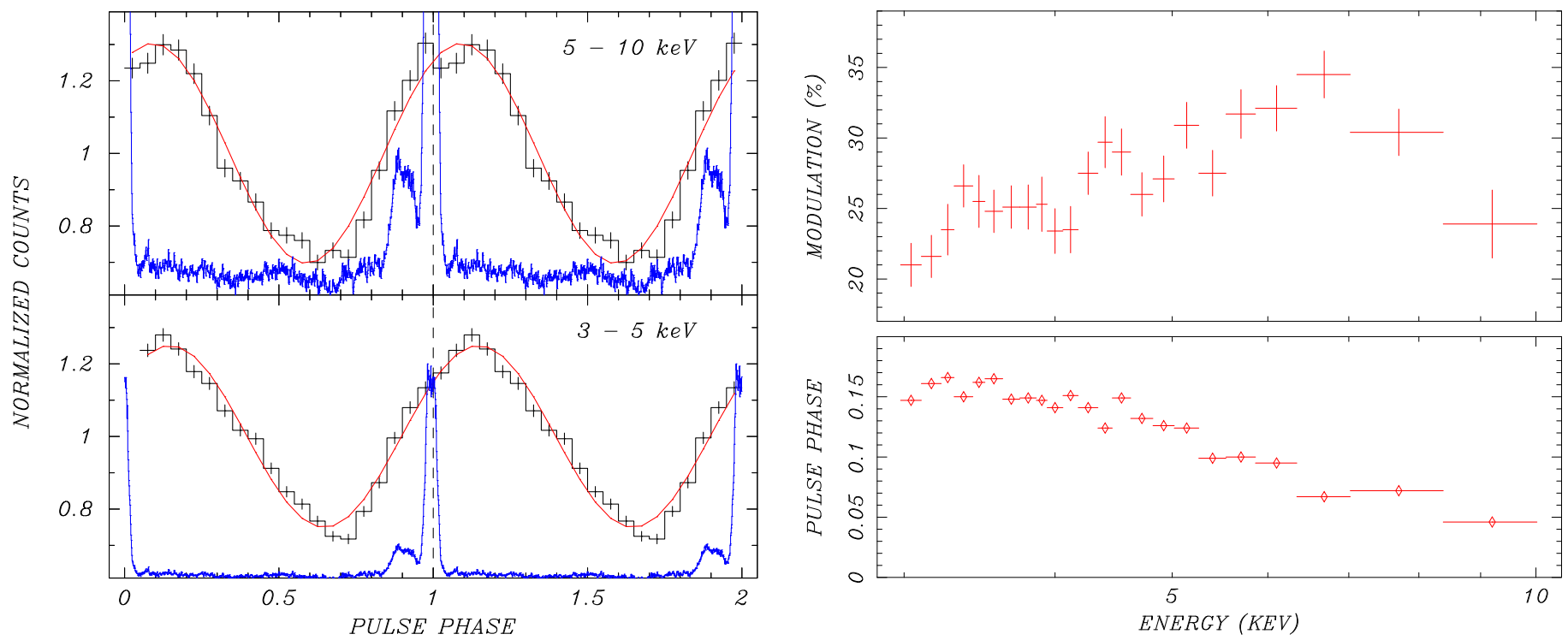

Figure 4. Left: NUSTAR background subtracted pulse profiles of XTE J1810-197 (black) in two energy bands folded on the radio ephemeris of M. Lower et al. (2019, in preparation), with the best-fit sinusoid model overlaid (red). Two cycles are shown for clarity. The X-ray observation started at MJD 58465.14 (2018 December 13). The blue trace is the radio pulse profile from the December 15 observation of Dai et al. (2019) using the Parkes telescope UWL receiver, with a center frequency of $2368 \mathrm{MHz}$ and bandwidth of $3328 \mathrm{MHz}$. Phase zero is defined as MJD 58467.943329663 (TDB). Two different scalings are shown to display the full dynamic range of the radio pulse. Right: X-ray pulse modulation (top panel) and phase (bottom panel) as a function of energy in uniform bins of $\approx 5000$ counts per fold. The slippage in phase with energy accounts for asymmetry in the broadband X-ray pulse profile.

Synthesis Telescope (UTMOST; Bailes et al. 2017). These were were used to develop an ephemeris that will be reported in M. Lower et al. (2019, in preparation). In Figure 4 (left), the X-ray photons were folded on the radio ephemeris. A radio pulse profile from Dai et al. (2019) that was obtained 2 days after the X-rays is shown in its absolute phase relation to the X-rays. The peak of the $\mathrm{X}$-rays lags the radio pulse by $\approx 0.13$ cycles.

Beginning in 2009, after pulsed radio emission turned off, the spin-down rate of XTE J1810-197 was very stable through 2018 (Camilo et al. 2016; Pintore et al. 2019). This enables us to extrapolate the Pintore et al. (2019) ephemeris to the epoch of the current observations. The predicted period at the NuSTAR epoch is 5.54146404(74), significantly larger than the NUSTAR measured value. This suggests that a large glitch occurred in conjunction with the (unobserved) onset of the outburst in late November. The radio ephemeris gives a more precise period than NUSTAR, and it suggests a glitch magnitude of $\Delta \nu / \nu=(4.52 \pm 0.15) \times 10^{-6}$, which is typical of large glitches observed in AXPs (Dib \& Kaspi 2014). This is probably a lower limit on the instantaneous glitch magnitude as it does not take into account any recovery due to an increase in period derivative between the epoch of the glitch and that of the first radio observation.

\section{Discussion}

During the long decay from its 2003 outburst, XTE J1810 -197 was monitored closely, revealing an evolving X-ray spectrum (Gotthelf \& Halpern 2007), its turnoff as a radio pulsar in 2008 (Camilo et al. 2016), its transition to X-ray quiescence in 2009 (Alford \& Halpern 2016; Pintore et al. 2016), and finally steady spin-down until 2018 (Pintore et al. 2019). The first detailed X-ray results for the 2018 outburst, reported herein, show similarities to this original event in terms of X-ray flux, spectrum, and pulse properties. However, the NUSTAR observation was obtained at an earlier phase in the outburst, within 2-3 weeks of the onset, compared to the 2003 event, when the first comparably useful XMM-Newton observations came 8-10 months after onset.

From spectral fits to XTE J1810-197 using the blackbody plus power-law model, the higher bolometric luminosity measured within weeks of the current outburst results in a larger blackbody area compared to those of the 2003 outburst. Both increases are consistent with catching the outburst at an earlier epoch. The blackbody temperature is also consistent with that reported for the 2003 outburst, when it remained constant for a year before fading linearly (Gotthelf \& Halpern 2007). However, for the two-blackbody model, one of the temperatures differs significantly from those reported for the 2003 outburst. Either the two temperatures are twice as hot as previously recorded, or an additional smaller, hotter emitting region is dominating the spectrum. The availability of NUSTAR broadband $\mathrm{X}$-ray image spectroscopy for the current outburst reveals a hard spectral component above $10 \mathrm{keV}$ not measurable during the prior outburst, either due to its disappearance a year into the event or for lack of sensitivity in the RXTE scan data at the time.

The measured hard spectral index favors the two-blackbody model yielding a value that is similar to that found for a typical magnetar, although the index measured using the alternative blackbody plus power-law model is not well constrained. However, two concentric thermal hot spots, as modeled for the 2003 outburst data (Perna \& Gotthelf 2008), is more consistent with the Beloborodov (2009) j-bundle picture in which the hot footpoint is surrounded by a warm X-ray emitting area. Future NuSTAR observations will help determine if the hard component is persistent.

The NUSTAR pulse properties differ from those observed during the previous outburst. Although the linear increase in modulation with energy is also evident at the earlier epoch, with roughly the same slope, the modulation for the newer data is half of that measured in 2003. This might be due to the larger area of the present emitting region, additional flux from the whole NS surface, or a different location of the emitting region during this early epoch of the 2018 outburst. The energy 
dependence of the modulation and phase shift of the pulse profile also suggests that the spectral components are currently not precisely coaligned. This may be transitory, as no phase shift with energy is evident following the 2003 outburst (Gotthelf \& Halpern 2005).

Unlike for the 2003 outburst, for which no regular radio monitoring was done until years after the X-ray event (Halpern et al. 2005; Camilo et al. 2006), intense radio pulsations heralded the onset of the current X-ray outburst. Given the sensitivity of the sparse VLA observations at the location of XTE J1810-197 compiled by Halpern et al. (2005), it is entirely possible that radio pulsations did commence in concert with the 2003 X-ray outburst. The current outburst behavior further supports such a scenario. Pulsar timing before and after the outburst also suggests that a glitch occurred in concert with the reactivation. This would not have been known of the 2003 outburst, as the pulsar was previously undiscovered. It is yet to be determined if the radio emission will fade smoothly, as for the 2003 outburst, or turn off at intervals during the X-ray decay, as is found for other transient radio magnetars.

Unlike ordinary pulsars, it is not known if radio emission from magnetars occurs on relatively stable, open dipole field lines, or on twisted, closed magnetic field-line j-bundles that conduct large currents and heat the NS surface to X-ray temperatures. Also, because outbursts can be triggered by crustal fractures, it is not clear that each new outburst must originate at the same location on the star. The radio phase relationship to the X-ray pulse, and the polarization swing of the radio pulse, may constrain the geometry and location of the emission regions as was attempted in Camilo et al. (2007b) and Kramer et al. (2007). More than 3 yr after the 2003 outburst, the phase alignment of the X-ray and radio pulse were compared in Camilo et al. (2007b), showing that their peaks almost coincide, with the $\mathrm{X}$-ray lagging the radio by $\approx 0.1$ cycles. With much better $\mathrm{X}$-ray statistics early in the current outburst, we have established that the X-ray pulse lags the radio peak by $\approx 0.13$ cycles, consistent with their behavior in the 2003 outburst. This suggests that the macroscopic geometry of the magnetic field associated with the emission did not change between outbursts.

One way to get a lag of the X-rays is for the radio emission to come from closed magnetic field lines on which there are currents heating the surface. In this case, the beaming of the radio emission along the curved magnetic field lines could account for the lag. Alternatively, if the radio emission comes from open field lines, at heights comparable to the radius of the light cylinder, then an X-ray lag would be expected from both light travel time effects and relativistic aberration. In the current outburst it is not yet possible to model the magnetic geometry and viewing angle using the rotating vector model of radio polarization. The early radio emission is highly erratic in degree and direction of polarization (Dai et al. 2019). If the radio flux persists, its polarization may settle down to allow the locations of the radio and X-ray emitting regions to be examined and compared to the results of the 2003 outburst. The role of open field lines versus closed field lines as the location of radio emission in magnetars, can be investigated well using XTE J1810-197, and is an important subject of exploration during the current outburst.

The NUSTAR mission is a project led by the California Institute of Technology, managed by the Jet Propulsion
Laboratory, and funded by the National Aeronautics and Space Administration. We thank the NUSTAR Director for timely approval of this observation of XTE J1810-197, and the NUSTAR Operations, Software, and Calibration teams for support with the execution and analysis of the observation. We thank the UTMOST team and the University of Sydney for maintaining the Molonglo Observatory. E.V.G. acknowledges NASA ADAP Grant NNX16AF30G. M.B. acknowledges ARC grant LF150100148. This research made use of the NuSTAR Data Analysis Software (NuSTARDAS) jointly developed by the ASI Science Data Center (ASDC, Italy) and the California Institute of Technology (USA). This research also made use of data and software provided by the High Energy Astrophysics Science Archive Research Center (HEASARC), which is a service of the Astrophysics Science Division at NASA/GSFC and the High Energy Astrophysics Division of the Smithsonian Astrophysical Observatory. We also acknowledge use of the Astronomer's Telegram (ATel) and the NASA Astrophysics Data Service (ADS).

\section{ORCID iDs}

E. V. Gotthelf (10 https://orcid.org/0000-0003-3847-3957

N. Kawai (i) https://orcid.org/0000-0001-9656-0261

M. E. Lower (iD https://orcid.org/0000-0001-9208-0009

M. Bailes (iD https://orcid.org/0000-0003-3294-3081

F. Camilo (10) https://orcid.org/0000-0002-1873-3718

H. Miyasaka (1D https://orcid.org/0000-0002-8074-4186

K. K. Madsen (iD https://orcid.org/0000-0003-1252-4891

\section{References}

Albano, A., Turolla, R., Israel, G. L., et al. 2010, ApJ, 722, 788

Alford, J. A. J., \& Halpern, J. P. 2016, ApJ, 818, 122

Arnaud, K. A. 1996, in ASP Conf. Ser. 101, Astronomical Data Analysis Software and Systems V, ed. G. Jacoby \& J. Barnes (San Francisco: ASP), 17

Bailes, M., Jameson, A., Flynn, C., et al. 2017, PASA, 34, 45

Beloborodov, A. M. 2009, ApJ, 703, 1044

Beloborodov, A. M. 2013, ApJ, 762, 13

Bernardini, F., Perna, R., Gotthelf, E. V., et al. 2011, MNRAS, 418, 638

Camilo, F., Cognard, I., Ransom, S. M., et al. 2007b, ApJ, 663, 497

Camilo, F., Ransom, S. M., Halpern, J. P., et al. 2006, Natur, 442, 892

Camilo, F., Ransom, S. M., Halpern, J. P., et al. 2016, ApJ, 820, 110

Camilo, F., Ransom, S. M., Halpern, J. P., \& Reynolds, J. 2007a, ApJL, 666, L93

Coti Zelati, F., Rea, N., Pons, J. A., Campana, S., \& Esposito, P. 2018, MNRAS, 474, 961

Dai, S., Lower, M. E., Bailes, M., et al. 2019, ApJ, submitted (arXiv:1902. 04689)

Desvignes, G., Eatough, R., Kramer, M., et al. 2018, ATel, 12285, 1

Dib, R., \& Kaspi, V. M. 2014, ApJ, 7484, 37

Durant, M., \& van Kerkwijk, M. H. 2006, ApJ, 650, 1070

Eatough, R. P., Falcke, H., Karuppusamy, R., et al. 2013, Natur, 501, 391

Enoto, T., Shibata, S., Kitaguchi, T., et al. 2017, ApJS, 231, 8

Esposito, P., Rea, N., \& Israel, G. L. 2018, arXiv:1803.05716

Gavriil, F. P., Kaspi, V. M., \& Woods, P. M. 2002, Natur, 419, 142

Gotthelf, E. V., \& Halpern, J. P. 2005, ApJ, 632, 1075

Gotthelf, E. V., \& Halpern, J. P. 2007, Ap\&SS, 308, 79

Gotthelf, E. V., Halpern, J. P., Buxton, M., \& Bailyn, C. 2004, ApJ, 605, 368 Gotthelf, E. V., Halpern, J. P., Grefenstette, B. W., et al. 2018, ATel, 12297, 1 Güver, T., Majid, W., Enoto, T., et al. 2019, ATel, 12484, 1

Halpern, J. P., \& Gotthelf, E. V. 2005, ApJ, 618, 874

Halpern, J. P., Gotthelf, E. V., Becker, R. H., Helfand, D. J., \& White, R. L. 2005, ApJL, 632, L29

Halpern, J. P., Gotthelf, E. V., Reynolds, J., Ransom, S. M., \& Camilo, F. 2008, ApJ, 676, 1178

Harrison, F. A., Craig, W. W., Christensen, F. E., et al. 2013, ApJ, 770, 103 Hori, T., Shidatsu, M., Ueda, Y., et al. 2018, ApJS, 235, 7

Ibrahim, A. I., Markwardt, C. B., Swank, J. H., et al. 2004, ApJL, 609, L21 
Kaspi, V. M., \& Beloborodov, A. M. 2017, ARA\&A, 55, 261

Kaspi, V. M., Gavrill, F. P., Woods, P. M., et al. 2003, ApJL, 588, L93

Kramer, M., Stappers, B. W., Jessner, A., Lyne, A. G., \& Jordan, C. A. 2007, MNRAS, 377, 107

Levin, L., Bailes, M., Bates, S., et al. 2010, ApJL, 721, L33

Lower, M., Bailes, M., Jameson, A., et al. 2018, ATel, 12288, 1

Lyne, A., Levin, L., Stappers, B., et al. 2018, ATel, 12284, 1

Madsen, K. K., Harrison, F. A., Markwardt, C. B., et al. 2015, ApJS, 220, 8

Matsuoka, M., Kawasaki, K., Ueno, S., et al. 2009, PASJ, 61, 999

Mihara, T., Negoro, H., Kawai, N., et al. 2011, PASJ, 63S, 623

Mihara, T., Negoro, H., Kawai, N., et al. 2018, ATel, 12291, 1
Minter, A. H., Camilo, F., Ransom, S. M., Halpern, J. P., \& Zimmerman, N. 2008, ApJ, 676, 1189

Mori, K., Gotthelf, E. V., Dufour, F., et al. 2014, ApJ, 793, 88

Negoro, H., Kohama, M., Serino, M., et al. 2016, PASJ, 68, S1

Perna, R., \& Gotthelf, E. V. 2008, ApJ, 681, 522

Pintore, F., Bernardini, F., Mereghetti, S., et al. 2016, MNRAS, 458, 2088

Pintore, F., Mereghetti, A., Esposito, P., et al. 2019, MNRAS, 483, 3832

Rybicki, G. B., \& Lightman, A. P. 1986, Radiative Processes in Astrophysics (New York: Wiley-VCH)

Shannon, R. M., \& Johnston, S. 2013, MNRAS, 435, L29

Woods, P. M., Kouveliotou, C., Gavriil, F. P., et al. 2005, ApJ, 629, 985 